\title{
Review
}

Zhenzhe Ma, Peiyan Li, Sai Chen* and Xiaojun Wu*

\section{Optical generation of strong-field terahertz radiation and its application in nonlinear terahertz metasurfaces}

\author{
https://doi.org/10.1515/nanoph-2021-0714 \\ Received November 15, 2021; accepted January 16, 2022; \\ published online January 31, 2022
}

\begin{abstract}
Extremely nonlinear terahertz (THz)-matter interactions and applications have positioned themselves as the next frontier in quantum information, nonlinear optics, and particle acceleration. However, the absence of free-space highly intense $\mathrm{THz}$ sources and the diffraction limit, which prevents $\mathrm{THz}$ waves from being concentrated to the nanoscale scale, are inhibiting the growth of extreme THz. To address this difficulty, suitably extremely concentrated $\mathrm{THz}$ sources are being produced, while (non-)resonant artificial metastructures are being widely used to enhance local fields, resulting in deepsubwavelength $\left(<\lambda / 10^{3}\right)$ confinement of highly enhanced $\mathrm{THz}$ fields in micro-/nano-gaps. We discuss solid-state stable sources of intense $\mathrm{THz}$ radiation generated by femtosecond lasers in this Review, with a special emphasis on the lithium niobate-based tilted pulse front approach and the nonlinear THz metasurfaces allowed by it. Finally, we forecast the field's future directions in extreme THz research.
\end{abstract}

Keywords: extreme $\mathrm{THz}$; lithium niobate; local field enhancement; nonlinear metasurface; strong-field $\mathrm{THz}$ radiation; tilted pulse front.

\section{Introduction}

Terahertz $(\mathrm{THz})$ radiation $(0.1-30 \mathrm{THz})$, as indicated in Figure 1, is between the microwave and infrared electromagnetic frequency bands, with photon energies close

\footnotetext{
* Corresponding authors: Sai Chen and Xiaojun Wu, School of Electronic and Information Engineering, Beihang University, Beijing 100191, China, E-mail: saichen@buaa.edu.cn (S. Chen), xiaojunwu@buaa.edu.cn (X. Wu). https://orcid.org/0000-00032647-4303 (S. Chen). https://orcid.org/0000-0001-8737-6286 (X. Wu)

Zhenzhe Ma and Peiyan Li, School of Electronic and Information Engineering, Beihang University, Beijing 100191, China, E-mail: zhenzhema@buaa.edu.cn (Z.Ma), peiyanli@buaa.edu.cn (P. Li)
}

to the Fermi level and peak electric and magnetic field intensities around or above $\mathrm{MV} / \mathrm{cm}$ and Tesla, respectively [1]. Additionally, its picosecond/sub-picosecond time resolution enables a wide variety of ultrafast spectroscopic and imaging applications. As a result, it can function as a unique cold light source, revealing a new unexplored realm of the fascinating interaction of light and matter [2-25].

$\mathrm{THz}$ frequencies correspond to the intrinsic phonon and magnon vibrations of a large number of strongly correlated systems. Thus, intense $\mathrm{THz}$ fields at a certain frequency can stimulate lattice resonance coherently and resonantly, thereby inducing novel electronic structures, discovering new physics, and obtaining new states. It has spawned a new field of research known as lightwave quantum electronics, which is geared toward quantum information processing applications. Additionally, THz pulses can align molecule orientation and hence regulate a large number of catalytic events in chemical engineering [26-29]. $\mathrm{THz}$ pulses with a strong field can flip electron spin and enable nonlinear spin control, laying the groundwork for future ultrafast spintronic devices [21, 30-36]. When combined with scanning probe technology, strong-field $\mathrm{THz}$ pulses can generate a tunneling current at the tip of the scanning tunneling microscope (STM), overcoming the diffraction limit of the $\mathrm{THz}$ lightwave and providing a new potent instrument for the state management of novel nanoscale materials [6, 37-41]. Strong-field THz pulses have the ability to accelerate, compress, and manipulate electron micro-bunches in several dimensions, which is predicted to result in the development of table-top miniaturized $\mathrm{THz}$ accelerators for use in tiny attosecond X-ray sources [42-53]. Besides, intense $\mathrm{THz}$ has been used to examine the biological effects of $\mathrm{THz}$ fields in biological applications [54-56].

Light-matter regulation falls within the topic of nonlinear optics in terms of optical science and technology. At lower frequencies, the majority of nonlinear phenomena in the $\mathrm{THz}$ regime are optical responses, such as nonlinear transmission, reflection, and absorption in materials driven by strong field $\mathrm{THz}$ pulses. Nonlinear $\mathrm{THz}$ 


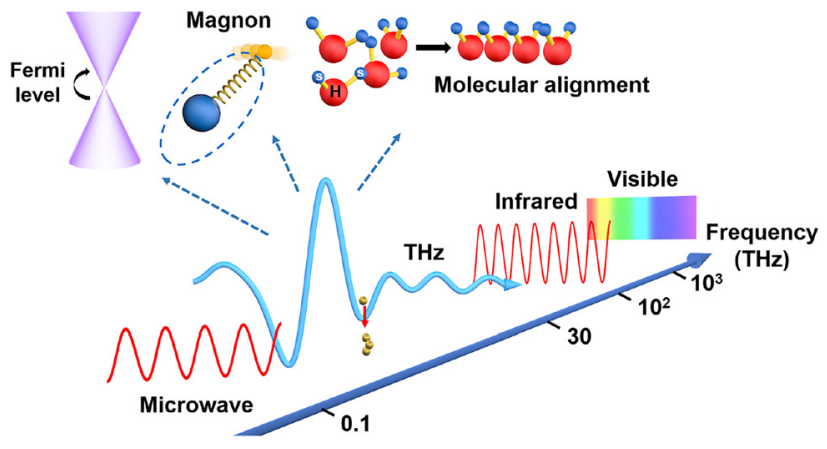

Figure 1: The location of $\mathrm{THz}$ frequency range in the electromagnetic spectrum is between microwave and infrared light. THz photon energy is around the Fermi level and its frequency corresponds to some magnon, phonon, or molecular vibration and rotation energy level.

research differ slightly from those conducted in the optical frequency range [21]. The incident light oscillates with the electric and magnetic degrees of matter in conventional nonlinear optics, imposing forces on the electrons (charge $e$, where $e$ is the elementary charge) and the ions (charge $q=-e$ ), thus inducing an electric-dipole density (polarization) $\boldsymbol{P}$, which is accompanied by a polarized current density $\mathrm{d} \boldsymbol{P} / \mathrm{d} t$. At the optical wavelength range, oscillations occur at the femtosecond level, where the Coulomb force $\boldsymbol{F}$ dominates the interaction of light and matter. When the incident light is $\boldsymbol{E}, \boldsymbol{F}$ can be written as $\boldsymbol{F}=q \boldsymbol{E}$. As a result, the nonlinear phenomena is predominantly associated with charge distributions (polarization). While $\mathrm{THz}$ field oscillations have a significantly longer duration than optical radiation, nonlinear polarization is no longer dominating. Due to the fact that its specific frequencies are capable of driving low-frequency motions such as molecular rotation and crystal lattice vibrations via coupling to ionic, electronic, or spin degrees of freedom (see Figure 2), it can resonantly excite these specific modes in the presence of strong-field $\mathrm{THz}$ lightwaves. Furthermore, non-resonant stimulation can cause a variety of nonlinear reactions. When the $\mathrm{THz}$ peak field amplitude $\boldsymbol{E}_{\max }$ at $1 \mathrm{THz}$ is above $0.3 \mathrm{MV} / \mathrm{cm}$, the ponderomotive energy
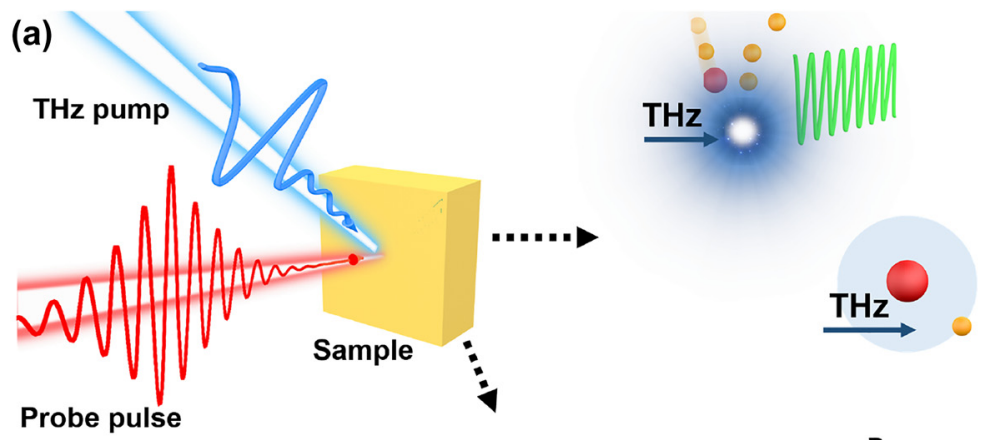

Probe pulse
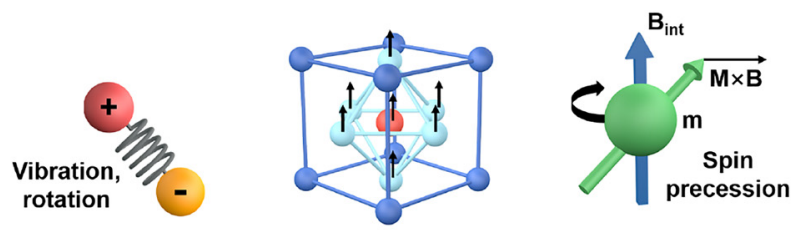

(b)
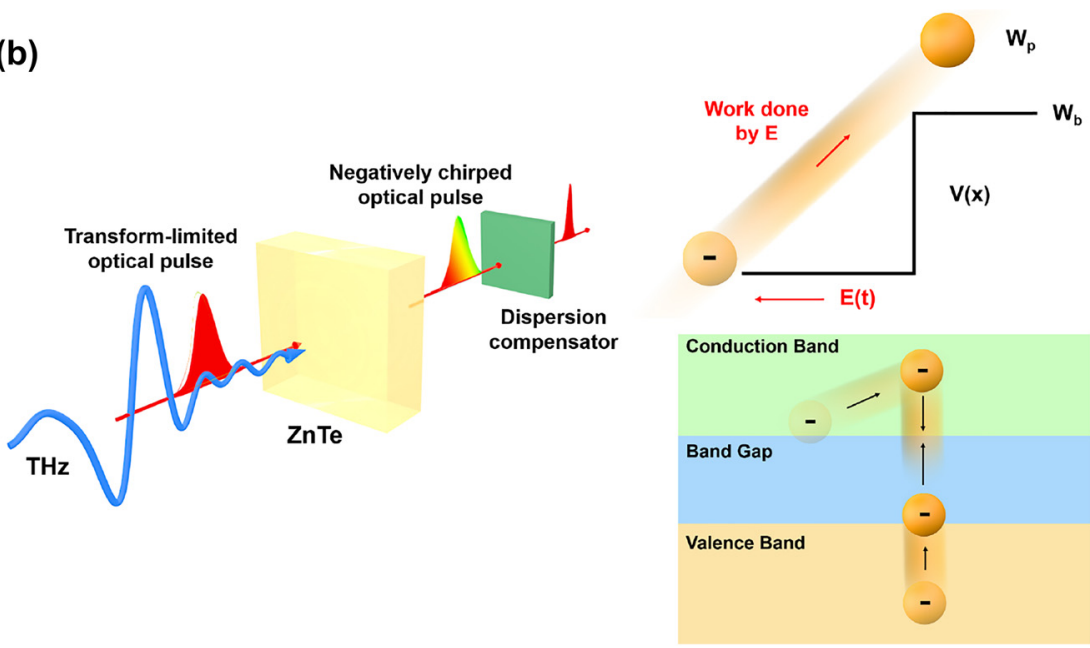

Figure 2: The interactions between strong $\mathrm{THz}$ waves and materials.

(a) Resonant (such as molecular rotation and crystal lattice vibrations, crystal lattice vibrations, coupling to ionic, electronic, or spin degrees of freedom, etc.) and (b) nonresonant (such as field ionization and impact ionization) interaction between strong $\mathrm{THz}$ waves and materials. Reprinted with permission from Kampfrath et al. [21] (C)Springer Nature Limited (2013) and katayama et al. [71] (C)American Physical Society (2012). 
$W_{q}$ can reach $1 \mathrm{eV}$ based on the following ponderomotive energy equation $W_{p}=\frac{e^{2} \boldsymbol{E}_{\max }{ }^{2}}{4 m^{*} \omega^{2}}$, where $m^{*}$ and $\omega$ denote effective mass and angular frequency of $\mathrm{THz}$ field respectively. This energy is already greater than the ionization energy of an impurity or an excision in a semiconductor, which can result in impact ionization [57-64] and field tunneling effects [39-41, 59, 65-70].

However, the absence of cost-effective, highly efficient, polarization-manipulated strong-field $\mathrm{THz}$ sources equivalent to those found in other sections of the electromagnetic spectrum is inhibiting the spread of nonlinear $\mathrm{THz}$ optics [72-81]. In comparison to ultrashort and super-strong femtosecond laser sources operating at visible and near-infrared frequencies, the single pulse energy, peak field strength, and peak power of ultra-strong $\mathrm{THz}$ radiation remain significantly lower. Additionally, several tens of $\mathrm{mJ} \mathrm{THz}$ pulses with extremely reliable performance are required to develop tiny $\mathrm{THz}$ electron accelerators [82]. The significantly greater peak field strength of $\mathrm{THz}$ pulses with tunable polarization states and defined temporal structuring has not yet been realized for extreme $\mathrm{THz}$ science research. As a result, there is still much space for improvement in the performance of strong-field $\mathrm{THz}$ sources. How to achieve leapfrog progress from weakfield passive detection to strong-field triggered treatments also requires extremely intense $\mathrm{THz}$ fields. These potentially major uses motivate us to design $\mathrm{THz}$ sources that are extremely robust, stable, and user-friendly. Certain questions, such as how to generate more powerful $\mathrm{THz}$ radiation, how to increase the efficiency of optical-to- $\mathrm{THz}$ energy conversion, and what the strongest $\mathrm{THz}$ laser that humans can create, are also extremely interesting scientific and technical challenges.

$\mathrm{THz}$ frequency, single-pulse energy (photon counts per pulse), energy conversion efficiency, peak electric and magnetic field intensity, and temporal waveform shape are the most important factors in several applications. Apart from typical accelerator-based $\mathrm{THz}$ radiation sources, the majority of sources for high field $\mathrm{THz}$ in free space are based on femtosecond laser pulse interaction with matter. As demonstrated in Table 1, laser-driven intense $\mathrm{THz}$ sources can be classified as photoconductive antennas, plasma in solids, gases, and liquids, and optical rectification [76]. It is noted that $\mathrm{THz}$ radiation efficiency is typically calculated by directly dividing the $\mathrm{THz}$ single pulse energy by the pumped laser energy applied to the emitting material. However, there is no consensus on the standard for calibrating $\mathrm{THz}$ energy probes. As a result, electro-optical (EO) sampling or single-shot diagnosis are used to determine the $\mathrm{THz}$ peak field intensity indirectly and thus to
Table 1: Record numbers for high-field THz sources.

\begin{tabular}{lll}
\hline THz sources & Max pulse energy & Efficiency (\%) \\
\hline $\mathrm{OR}^{*}$ in $\mathrm{LiNbO}_{3}$ & $1.4 \mathrm{~mJ}[79]$ & $3.8[85]$ \\
$\mathrm{LiNbO}_{3} \mathrm{PPLN}$ & $0.6 \mathrm{~mJ}[72]$ & $1.0[72]$ \\
$\mathrm{OR}^{*}$ in organic crystal & $0.9 \mathrm{~mJ}[86]$ & $3.0[86]$ \\
$\mathrm{OR}^{*}$ in $\mathrm{ZnTe}$ & $14 \mu \mathrm{J}[87]$ & $0.7[87]$ \\
Conventional accelerators & $600 \mu \mathrm{J}[88]$ & $\mathrm{n} / \mathrm{a}$ \\
Photoconductive antenna & $11 \mu \mathrm{j}[89$ & $1.6[90]$ \\
Laser-induced sheath field & $0.7 \mathrm{~mJ}[91]$ & $\mathrm{n} / \mathrm{a}$ \\
Plasma in air and gases & $0.19 \mathrm{~mJ}[73]$ & $2.36[73]$ \\
Laser-solid foil interactions & $202 \pm 100 \mathrm{~mJ}[92]$ & $0.29[92]$ \\
Liquids (water) & $76 \mu \mathrm{j}[78]$ & $0.3[78,93]$ \\
\hline
\end{tabular}

correct the detected THz energy. Even so, when ultra-highfrequency THz waves are detected with an EO crystal, linear EO effects frequently exhibit over rotation, whereas nonlinear effects are easily induced by a strong electric field, resulting in measurement error. As a result, novel diagnostic techniques for developing extremely intense $\mathrm{THz}$ sources must be investigated. The intuitive option is to generate stronger $\mathrm{THz}$ radiation in free space, while the other is to develop a mechanism to realize local field augmentation to match application requirements that are currently unattainable due to the $\mathrm{THz}$ radiation's limiting electric field [57, 60, 61, 83, 84], as illustrated in Figure 3.

The core concept behind local field enhancement techniques in artificial structures is based on lightning rod and antenna effects. THz fields are confined and localized within metal micro-/nano-gaps, producing resonant or non-resonant field enhancement responses. If artificial

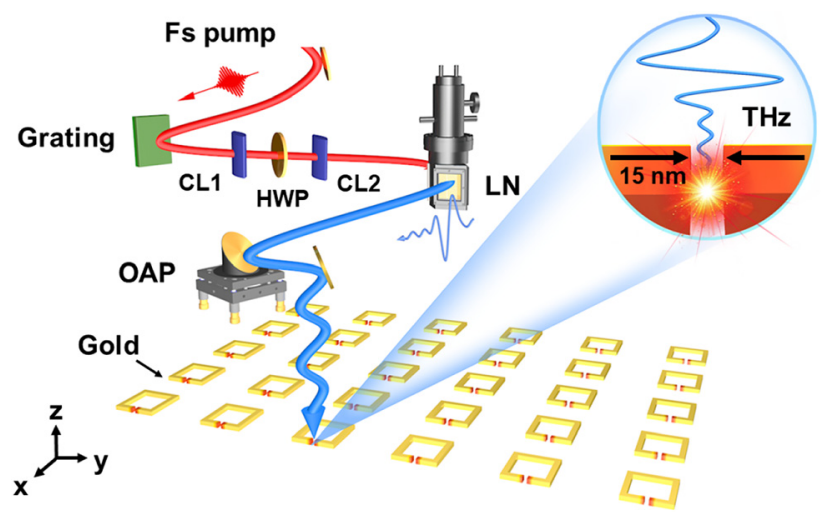

Figure 3: Schematic diagram for achieving high field $\mathrm{THz}$ radiation based on lithium niobate or local field enhancement in metasurfaces.

CL: cylindrical lens; HWP: half-wave plate; LN: lithium niobate; OAP: off-axis parabolic mirror. Reproduced with permission from Dong et al. [61] C)Wiley-VCH GmbH (2021). 
structures are delicately created at specific frequencies, they can serve two critical functions. One is used to boost the local field for THz pumping, while the other is used for sensitive probing. This method was widely used in the early years of strong-field $\mathrm{THz}$ science and applications, and numerous field-induced impact ionization and tunneling events were identified, culminating in the production of non-equilibrium conductivity in micro-/nanogaps. This approach allows for localized enhancements of the $\mathrm{THz}$ field strength of many orders of magnitude, causing insulator-to-metal phase transitions, nonlinear spin switching, and ultrafast photoluminescence emission, among others. We focus on the lithium niobate crystal-based tilted pulse front approach via optical rectification for generating $\mathrm{mJ}$ or even tens of $\mathrm{mJ}$ free-space $\mathrm{THz}$ radiation in this paper, as well as the creation of nonlinear $\mathrm{THz}$ metasurfaces enabled by lithium niobate $\mathrm{THz}$ sources.

\section{Intense THz radiation from lithium niobate}

When femtosecond laser pulses interact with solids, liquids, gases, or plasma, strong-field $\mathrm{THz}$ radiation can be generated (see Figure 4). Different THz radiation processes exhibit distinct characteristics. While some sources of intense $\mathrm{THz}$ radiation are less stable, the emission mechanism is extremely interesting to study. It is not only fascinating in terms of radiation mechanism, but also extremely useful in terms of technology and real-world applications for solid-state $\mathrm{THz}$ sources. $\mathrm{THz}$ radiation mechanisms in solid-state strong-field sources can be classified as optical rectification (OR), difference frequency generation (DFG), and the inverse spin Hall effect in ferromagnetic metal/heavy metal heterostructures [94-97]. When the radiation single pulse energy, optical-to- $\mathrm{THz}$ efficiency, field strength, stability, and expandability are all taken into account, OR and DFG are preferable [86, 98-100]. OR in lithium niobate for high field $\mathrm{THz}$ generation has become ubiquitous in low-frequency applications. What's more, in 2021, lithium niobate will achieve record levels of single pulse energy with $1.4 \mathrm{~mJ}, 800 \mathrm{~nm}$-to- $\mathrm{THz}$ energy conversion efficiency of $0.7 \%$, and focused electric field strength $>6.4 \mathrm{MV} / \mathrm{cm}$.

Indeed, the first $\mathrm{THz}$ pulse was generated from lithium niobate in 1971 [101]. Due to its mature growth technology, lithium niobate can be grown in large sizes and has a high second-order nonlinear coefficient. Additionally, when doped with $\mathrm{MgO}$, lithium niobate crystals

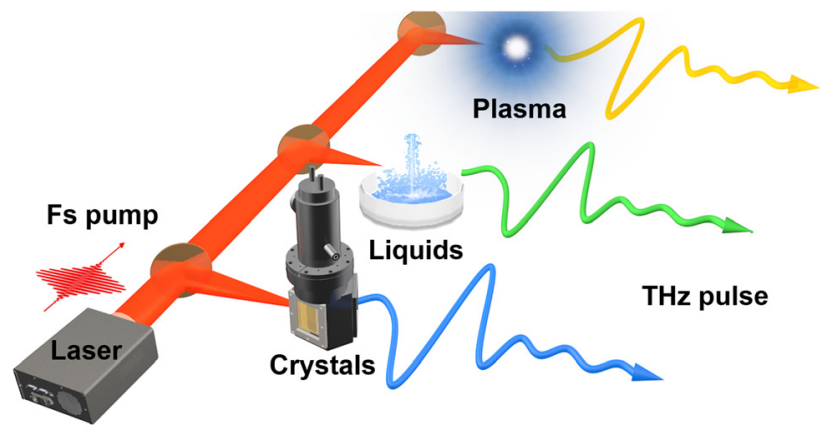

Figure 4: Strong field THz radiation based on nonlinear crystals, liquids, and plasma.

can be used without causing damage on Joule-level high power femtosecond lasers. Lithium niobate has a bandgap of $4.0 \mathrm{eV}$, which allows for effective avoidance of multiphoton absorption during high-energy pumping. However, the phase matching problem and strong linear absorption for $\mathrm{THz}$ radiation preclude lithium niobate from being a useful THz source. Fortunately, the tilted pulse front technique was demonstrated successfully in 2002, resolving the phase mismatching problem [102]. Since then, development of strong-field $\mathrm{THz}$ sources based on lithium niobate has accelerated. Between 2002 and 2021, the tilted pulse front technique underwent nearly three stages of development, as illustrated in Figure 5. It is roughly equivalent to increasing efficiency, pursuing high single pulse energy, and optimizing focusing for high field strengths [103-106]. $\mathrm{THz}$ radiation of greater than $1 \mathrm{~mJ}$ has been experimentally demonstrated up to 2021. All efficiency, single pulse energy, and field strength have been increased by more than three orders of magnitude using various optimization techniques. Such tremendous progress is inextricably linked to collaborative experimental and theoretical innovation.

\subsection{Experimental optimization}

As depicted in Figure 3. A typical setup for a tilted pulse front consists of a pumping laser, a grating, an imaging system, and the lithium niobate crystal. As a result, optimization is primarily concerned with the three components. Optimizing pumping laser parameters entails determining the optimal pumping wavelength, pulse duration, spectrum distribution profile, and pumping energy [85, 109-113]. However, it is difficult to achieve significant improvement solely by optimizing a single parameter. In actual experiments, these parameters are interdependent 


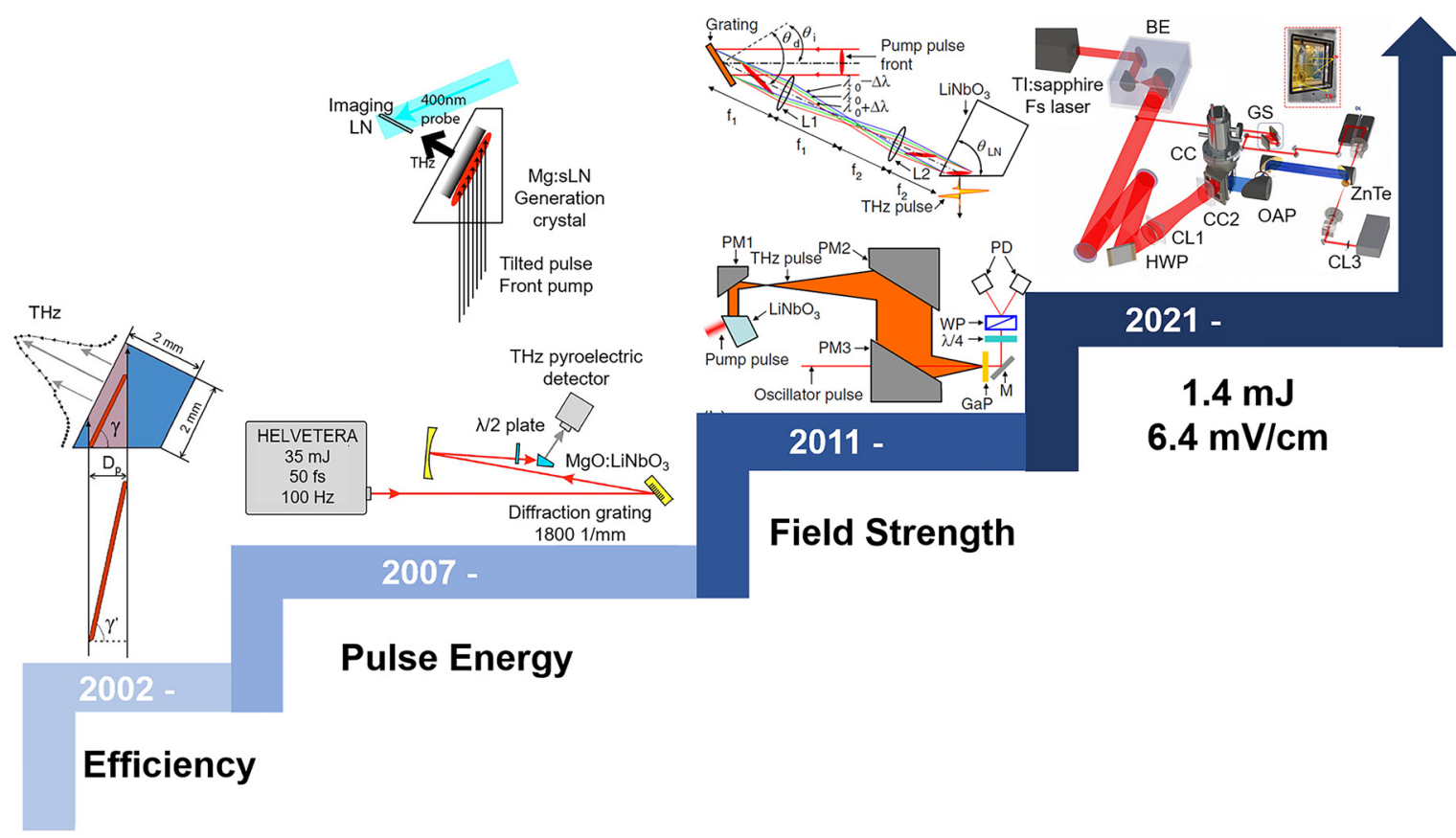

Figure 5: Evolution process for tilted pulse front technique based on lithium niobate. Reprint permission from [102] C)OPTICA (2002) [104]; (C)American Institute of Physics (2007) [107]; (C)OPTICA (2008) [108]; (C)American Institute of Physic (2011) [79]; CWiley-VCH GmbH (2021).

and frequently require cooperative regulation to produce acceptable experimental results. The primary challenges in optimizing the tilted pulse front optical path are selecting the dispersion component and imaging system. The majority of published results involved the use of gratings to tilt the pulse front of the pumping laser. Additionally, several novel structures have been proposed, including a structure formed by direct etching on the surface of a lithium niobate crystal or contact gratings [114-116]. Furthermore, because the efficiency of diffraction for tilting the pumping pulse fronts is not very high, these novel ideas have not yet gained widespread adoption. The imaging system is the other component. Numerous imaging techniques have been evaluated for increased efficiency, including single convex lenses, double convex lenses, and two cylindrical lenses [117]. Since the successful demonstration of a $1 \mathrm{MV} / \mathrm{cm}$ THz field strength with two cylindrical lenses, it has been established that this is the most efficient approach [108]. The nonlinear coefficient for crystal types, absorption for $\mathrm{THz}$ waves, and damage threshold are the primary considerations for crystal parameters. Lithium niobate has a relatively high congruent and stoichiometric ratio and has been widely used for strong-field $\mathrm{THz}$ generation. However, stoichiometric lithium niobate cannot be grown in large sizes suitable for moderate pumping energy in $\mathrm{kHz}$ femtosecond laser systems. Congruent lithium niobate crystals can be grown to extremely large sizes for extremely high power laser excitation. However, its linear absorption of $\mathrm{THz}$ waves generated within crystals must be overcome by cryogenically cooling [118]. When the generation crystals are cooled with liquid nitrogen, the efficiency of the radiated $\mathrm{THz}$ can be increased $[85,119]$. Further cooling the crystal to liquid helium temperature results in a negligible increase in $\mathrm{THz}$ efficiency [116]. In terms of damage threshold, $\mathrm{MgO}$ doping can enhance the crystal's performance, allowing for even more J-level femtosecond laser pumping. While mJ-level $\mathrm{THz}$ radiation from lithium niobate has been demonstrated successfully, future development will focus on 10-mJ or even Joule-level THz, providing free space THz fields larger than $10 \mathrm{MV} / \mathrm{cm}$ or even $\mathrm{GV} / \mathrm{cm}$ for extreme science and applications. The majority of driving laser systems used in high field $\mathrm{THz}$ generation are not purpose-built for it. To generate $\mathrm{THz}$ at the Joule level, we must design the pumping laser parameters and the tilted pulse front technique with care, as well as improve theoretical prediction.

\subsection{Theoretical progress}

The primary goal of tilted pulse front theory is to predict and guide experimental implementation of strong-field $\mathrm{THz}$ generation. Its evolution can be roughly classified into four stages: theory proposal, 1D, 2D-3D, and 3D +1 [120-124]. The theoretical prediction becomes increasingly 
close to the experimental results as the model is improved continuously. At the outset of the theory proposal, the phase matching wave vector relationship is transformed for the first time into the relationship between the pumping laser's group velocity and the $\mathrm{THz}$ wave's phase velocity. It illustrates that the two conditions are equal if only considering the first-order angular dispersion and regarding the $\mathrm{THz}$ frequency as an infinitesimal quantity compared with the optical frequency. In lithium niobate crystals, the group velocity of laser pulses is naturally larger than the phase velocity of $\mathrm{THz}$ waves, which is exactly why collinear phase matching cannot be achieved inside lithium niobate crystals. The titled pulse front technique utilizes the first-order angular dispersion to "decelerate" the group velocity of laser pulses until it synchronized with the phase velocity of $\mathrm{THz}$ waves. Consequently, phasing matching condition was satisfied in lithium niobate crystals approximately. It establishes an efficient phase matching scheme in lithium niobate crystals, enabling the experiment to be carried out successfully. The latter researchers derived several models to describe the $\mathrm{THz}$ generation process. The main idea of these models is to describe the second-order nonlinear optical processes inside lithium niobate crystals in continuous spectra, including the difference frequency generation of the $\mathrm{THz}$ radiation (Figure 6) and the secondorder interactions between the $\mathrm{THz}$ components and the pump laser pulses. Later, a one-dimensional model was developed that can qualitatively predict the generated $\mathrm{THz}$ energy, efficiency, and electric field strength in relation to the pumping laser and crystal parameters. The cascading process has been considered in light of the depletion of the pumping laser spectrum. As a result, the efficiency of optical-to-THz energy conversion should be constrained by the distortion of the pumping laser spectrum within lithium niobate crystals. The efficiency predicted theoretically has been reduced using this method. Then, the above model was extended to two spatial dimensions (2D) to depict the spatio-temporal variations of the optical pump pulses. Further, the 3D+1 numerical model thoroughly investigated the high field $\mathrm{THz}$ generation process in the tilted-pulse-front configuration, and it reminded us that the radiated $\mathrm{THz}$ pulses and their application experiments must account for significant spatial inhomogeneity. In the future, it will be critical to continue developing the theory for predicting the efficiency and $\mathrm{THz}$ properties of lithium niobate via the tilted pulse front technique for Joule-level $\mathrm{THz}$ generation. What's more intriguing is whether the tilted pulse front scheme can be inversely designed to achieve the desired $\mathrm{THz}$ characteristics. As a result, a successful combination of experimental and theoretical advances will accelerate the rapid advancement of nonlinear and extreme $\mathrm{THz}$ science and applications.

\section{Nonlinear THz metasurface}

Apart from generating free-space strong-field $\mathrm{THz}$ radiation for nonlinear $\mathrm{THz}$ investigations, another effective method of achieving higher electric fields is by enhancing local fields. By utilizing meta-structures such as metamaterials (metasurfaces), nano-tips, and nano-gaps, among others, one can enhance and localize the incident wave's electric field. Metamaterials, first proposed in the 1990s [125], are synthetic composites with unit cell sizes typically in the subwavelength range that can manipulate the amplitude, phase, and polarization of incident electromagnetic waves. Metasurfaces are a two-dimensional representation of their three-dimensional form, which is more easily realized and fabricated. The most attractive property of nonlinear $\mathrm{THz}$ metasurfaces is their strong ability to enhance and localize fields. Since the first

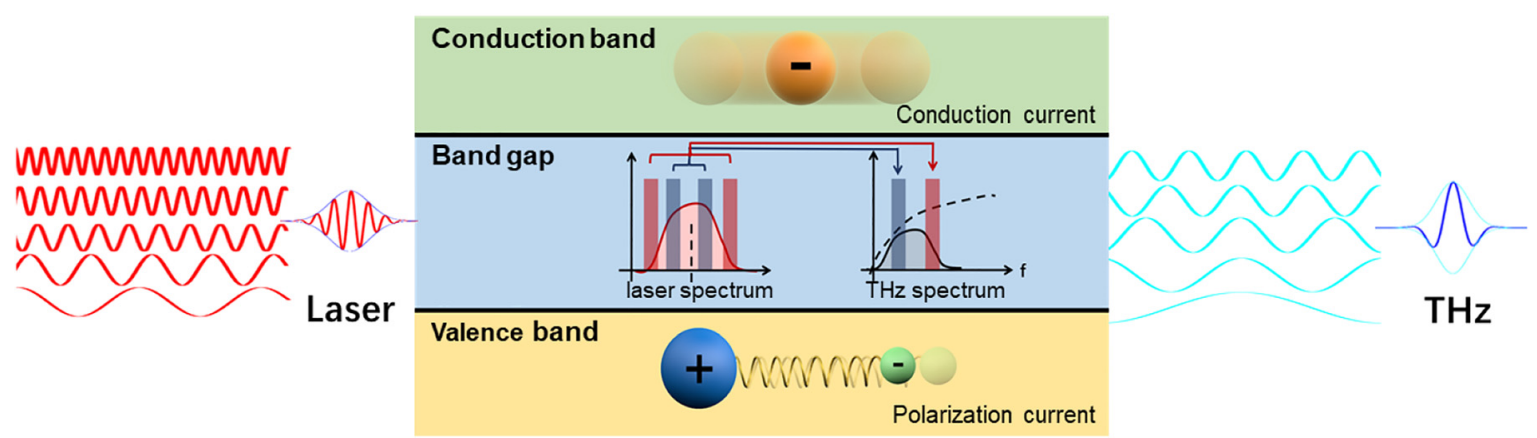

Figure 6: Physical picture of THz radiation from polarization and conduction currents, respectively. Difference frequency generation for THz radiation is inserted in the middle. 
experimental realization of a THz-induced vanadium dioxide phase transition in 2012 [84], nonlinear THz investigations have been strongly correlated with metasurfaces, providing an extremely effective scheme for studying nonlinear $\mathrm{THz}$ phenomena with a moderate free-space $\mathrm{THz}$ field strength of $\mathrm{kV} / \mathrm{cm}$. We review the development of the nonlinear $\mathrm{THz}$ metasurface in this section, with an emphasis on electric field enhancement.

\subsection{Resonant-enhanced nonlinear $\mathrm{THz}$ metasurface}

As previously stated, a split-ring resonator (SRR) metasurface (two-dimensional metamaterial) can be used to resonantly enhance picosecond and high-field $\mathrm{THz}$ pulses, thereby lowering the coulomb-induced potential barrier for carrier transport. The enhancement of the electric field is due to the inductance-capacitance (LC) resonance, which can be modeled using lumped equivalent circuits $[126,127]$. Numerous metasurface phenomena can be adequately explained using this model, and numerous related devices are demonstrated. As illustrated in Figure 7, the gap and spacer in both SRRs [57, 59, 64, 84, 128] and perfect absorbers [60] can be viewed as capacitance, while the metal structure can be viewed as inductance and resistance. At resonant frequencies, the E field is amplified and localized at the gap, inducing an intensitydependent response in the matter. Additionally, according to theory, the dielectric environments surrounding micro-/nano-gaps vary, which can be detected sensitively using a coherent $\mathrm{THz}$ probing beam.

The research on resonance-enhanced nonlinear $\mathrm{THz}$ metasurfaces is primarily concerned with structural engineering in order to increase the strength of the local $\mathrm{THz}$ field and then induce nonlinear responses in various substrate materials. The majority of reported investigations into meta-structure design and fabrication utilized microgap-enabled SRRs. These structures multiply the local field enhancement by several tens, which is not possible with traditional lithography. Nonetheless, such enhance- ments for free-space $\mathrm{THz}$ fields of several hundred $\mathrm{kV} / \mathrm{cm}$ can ensure that local fields exceed $\mathrm{MV} / \mathrm{cm}$. As a result, numerous nonlinear responses have been observed experimentally in phase transition materials and semiconductors $[57,59,61,63,84]$. The nonlinear behavior of phase transition materials such as $\mathrm{VO}_{2}$ film [84] is due to the insulator to metal transformation caused by strong field $\mathrm{THz}$ excitation. However, the mechanism of local field-induced nonlinearity is more complex in semiconductors, and can be classified as impact ionization [57, 59, 61, 63], intervalley scattering [57, 61], and interband tunneling [59]. To disentangle their contributions to $\mathrm{THz}$ nonlinearity, the doping level, incident field strengths, and substrate material types were all explored in detail. In 2013, the SRR metasurface (gap $\sim 2.2 \mu \mathrm{m}$ width) was deposited on $1.8 \mu \mathrm{m}$-thick doped $\left(1 \times 10^{16} \mathrm{~cm}^{-3}\right) \mathrm{GaAs}$ films. Intervalley scattering was observed at free-space $\mathrm{THz}$ peak fields of $\sim 20-160 \mathrm{kV} / \mathrm{cm}$ [57], which can reduce carrier mobility and enhance the SRR response due to a conductivity decrease in the doped thin film. When the $\mathrm{THz}$ field was greater than $160 \mathrm{kV} / \mathrm{cm}$, the increased electric field resulted in impact ionization, which increased carrier density and conductivity. Intriguingly, in 2014, SRR metamaterials (gap $\sim 2.5 \mu \mathrm{m}$ width) were fabricated on an undoped GaAs substrate, where the THz field strength can reach the ponderomotive energy in the $\mathrm{keV}$ range. Nondestructive quasistatic interband tunneling and impact ionization were demonstrated experimentally by observing ultra-broadband near-infrared and visible interband luminescence induced by the enhanced $\mathrm{THz}$ local field with $\sim 10 \mathrm{MV} / \mathrm{cm}$ [59], as illustrated in Figure 8. Except these non-tunable resonantly enhanced nonlinear $\mathrm{THz}$ metasurfaces, an electrically active nonlinear $\mathrm{THz}$ SRR metamaterial $(\sim 1 \mu \mathrm{m}$ width) was proposed [129]. External DC bias can be used to control the resonance's strength and nonlinearity, resulting in the nonlinear $\mathrm{THz}$ metasurface functionalization. In short, nonlinear $\mathrm{THz}$ phenomena can be investigated without being completely constrained by the free-space $\mathrm{THz}$ field strength using resonant metasurfaces with micrometer gaps.

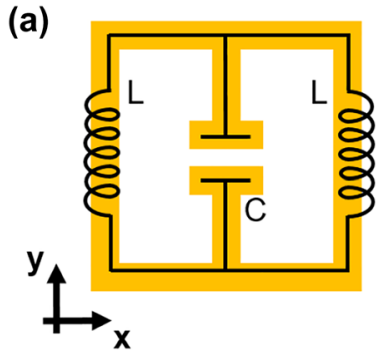

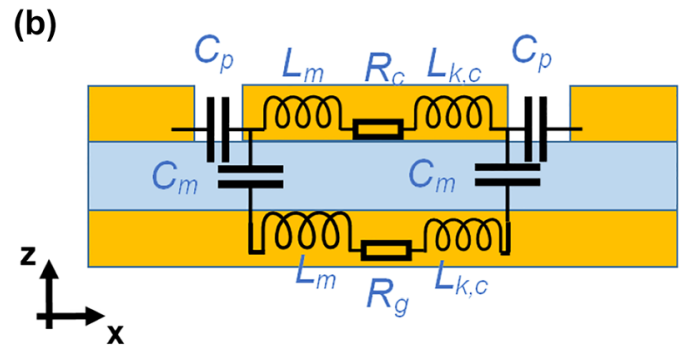

Figure 7: Classical lumped equivalent-circuit model for (a) split-ring resonators and (b) perfect absorber metasurfaces. 


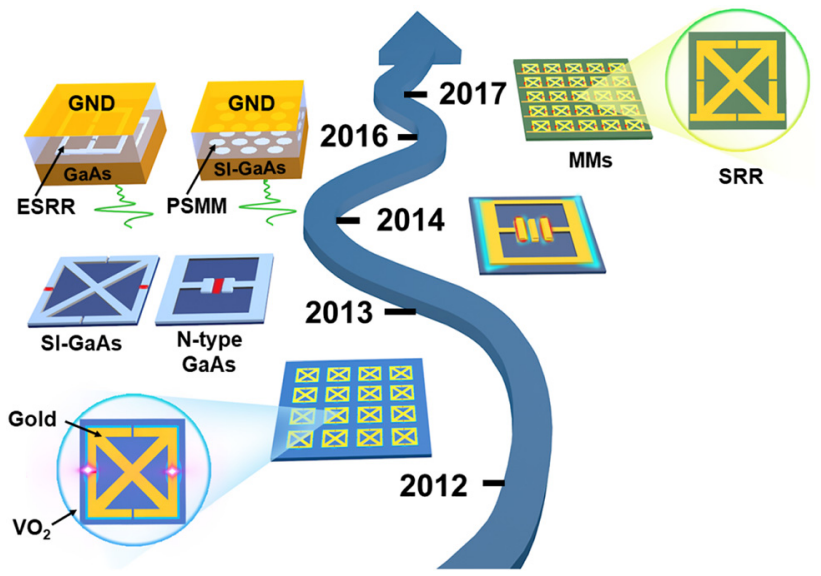

Figure 8: Timeline of the development on THz split-ring resonator nonlinear metasurfaces. Reproduced with permission from [21] (C) Springer Nature Limited (2013) [57, 59]; (C)American Physical Society (2014) [60]; COPPIICA (2016) [129]; C)American Institute of Physics (2017).

\subsection{Non-resonant nonlinear $\mathrm{THz}$ metasurfaces}

Resonant metasurfaces have been proved to be an effective way to enhance the $\mathrm{THz}$ electric field. However, the resonance can only enhance the localized field for certain frequencies. For broadband high field $\mathrm{THz}$ pulses, non-resonant metasurfaces are proposed for general local field enhancement. Normally, such kinds of structures, as illustrated in Figure 9, are constructed by one-dimensional subwavelength grating or nano-slot metasurfaces $[65,68$, $70,130]$, Metasurfaces with resonant properties have been

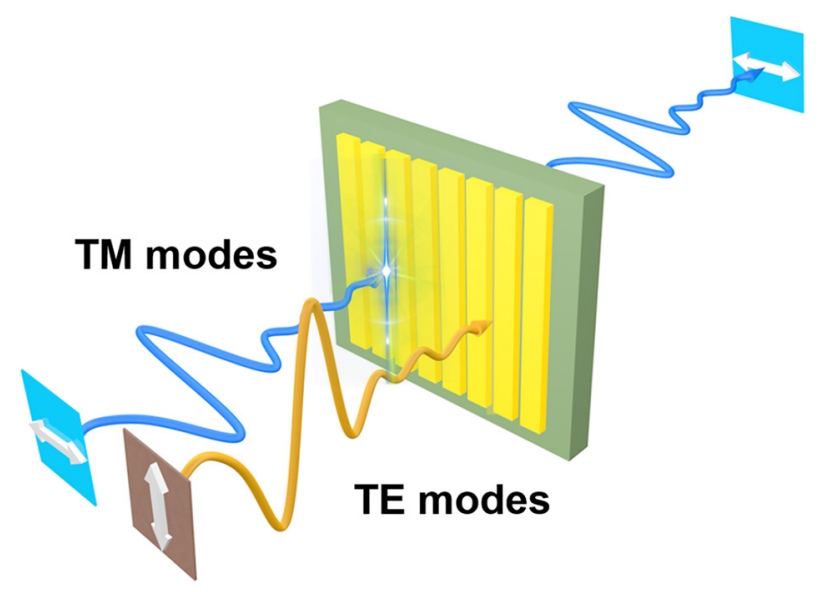

Figure 9: The schematic of the nano-slot metasurfaces (one-dimensional subwavelength grating). shown to be an effective method of increasing the THz electric field. However, resonance can enhance the localized field only for a limited range of frequencies. Non-resonant metasurfaces are proposed for general local field enhancement with broadband high field $\mathrm{THz}$ pulses. Typically, such structures are constructed using one-dimensional subwavelength gratings or nano-slot metasurfaces that support transmitting TM mode but not TE mode. As a result, the only way for $\mathrm{THz}$ waves to transmit through this grating is through the gaps or slots. Local field enhancement is not frequency dependent in this case, but is highly dependent on the gap size and duty ratio.

As a result, for the gap parameters, micro-, nano-, and even angstrom-scale values can be used to achieve extremely effective local field enhancement. More intriguingly, a fabrication technique called "plug-and-peel" metal patterning $[68,130]$ has been developed to enable the creation of slots of varying sizes. In Figure 10, a graphene atomic layer is shown acting as an angstrom-sized plug. When it is peeled away, nano-slots between the metal stripes form, resulting in metal-nano-spacer-metal metasurfaces [130]. The slot width can be reduced to $0.3 \mathrm{~nm}$ using this method, resulting in a $5 \times 10^{7}$ times. fold increase in the localized field enhancement factor. Due to the extremely large ponderomotive energy, such high fields can induce electron tunneling. The presence of obvious $\mathrm{THz}$ nonlinearity is detected. Additionally, insulators such as $\mathrm{Al}_{2} \mathrm{O}_{3}$ are used to replace graphene, and numerous nonlinear responses in the $\mathrm{THz}$ regime are observed.

Similarly, metal nano-slots have been used to enhance the $\mathrm{THz}$ field and further stimulate the luminescence of CdSe-CdS core-shell quantum dots [70]. When the $\mathrm{THz}$ driving field is increased to $100 \mathrm{kV} / \mathrm{cm}$, the quantum dots begin to glow and become visible to the naked eye (Figure 11). The ponderomotive energy is calculated to be greater than $0.5 \mathrm{eV}$ in this case, with an unusually high and rapid modulation of the bandgap.

In short, non-resonant metasurfaces can also effectively enhance the electric field, and numerous nonlinear $\mathrm{THz}$ responses have been achieved as a result of the high ponderomotive energy. Particularly when the gap is on the nanometer scale, the electric field can be magnified by a factor of five. Recalling what we discussed previously about resonant metasurfaces, an intriguing question is whether these two types of enhancement structures can be effectively combined to investigate $\mathrm{THz}$ nonlinearity. The majority of gap widths in resonant metasurfaces are greater than $1 \mu \mathrm{m}$, and the localized (in-gap) electric field enhancement is typically less than 50 times. The primary difficulty 
(a)

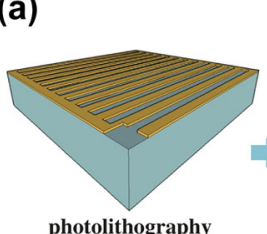

photolithography

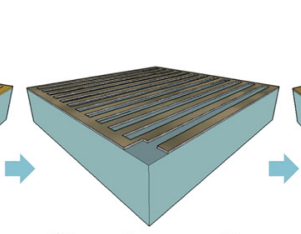

SL graphene growth
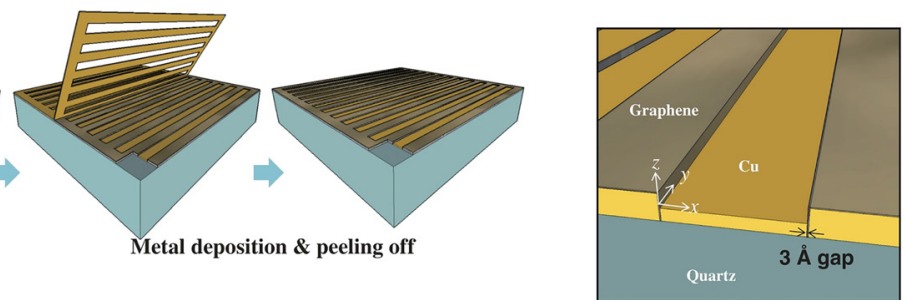

(b)

Pattern and ALD

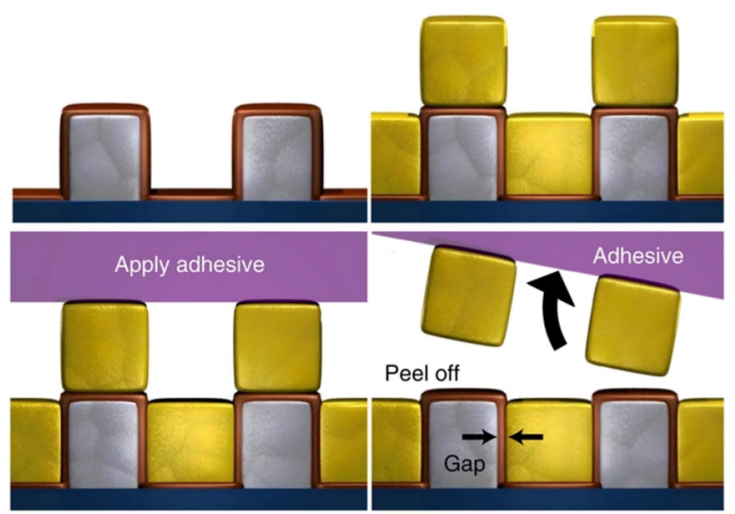

(c)
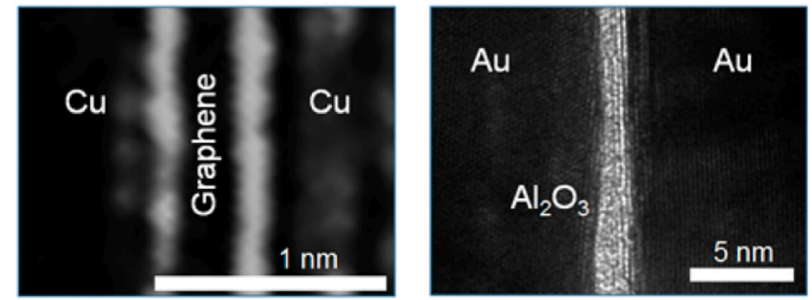

Figure 10: Nonlinear THz nano-slots based on "plug-and-peel" metal patterning technique for observing nonlinear phenomena.

(a) Graphene [130], (b) $\mathrm{Al}_{2} \mathrm{O}_{3}[68,131]$. (c) SEM images of the metasurfaces. Reproduced with permission from [130] (C)American Physical Society (2015) [68]; (C)American Chemistry Society (2015) [131]; C)Springer Nature Limited (2013).

(a)

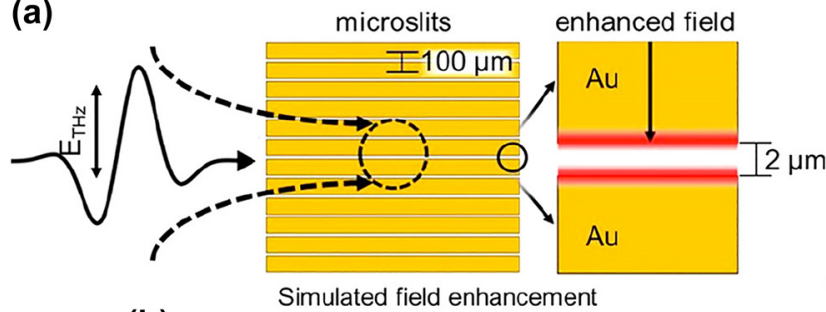

(b)

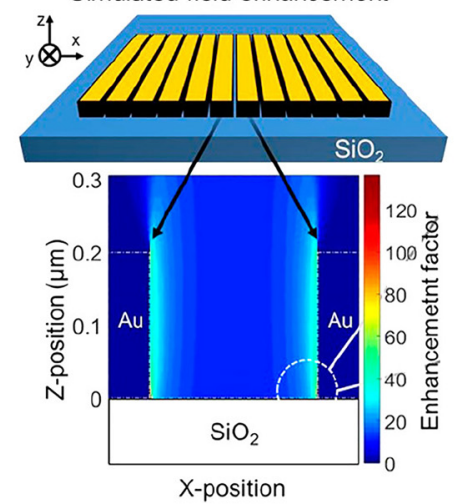

(c)

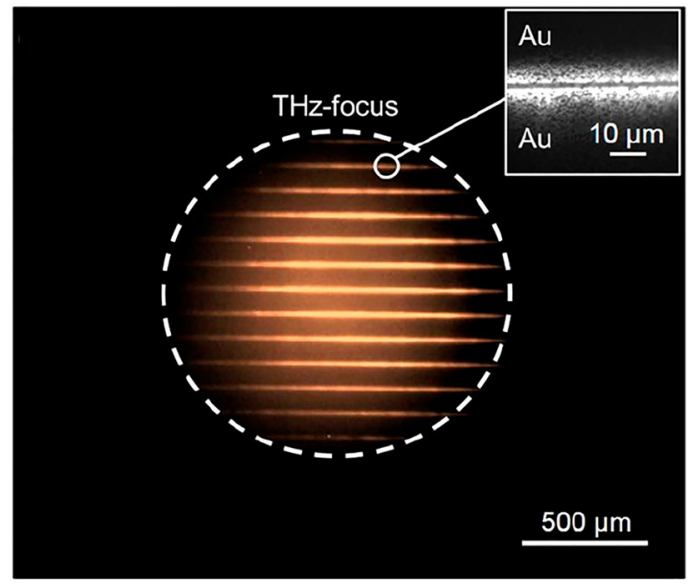

Figure 11: Strong field THz induced quantum dots luminescence [70].

(a) The THz field was enhanced in the $2 \mu \mathrm{m}$ capacitive gaps between the gold lines, where quantum dots were deposited over; (b) simulation of microslit THz near-field enhancement; (c) focusing THz pulses onto samples generates a visible light image. Reproduced with permission from Pein et al. [70] (C)American Chemistry Society (2017).

in combining resonant and non-resonant metasurfaces is narrowing the gap size in resonant metastructures with large sample sizes to the nanometer scale. To accomplish this, a THz SRR metasurface with a nano-gap [61] is created by combining traditional lithography and a novel procedure (Figure 12). 
Using this nano-gap SRR metasurface, nonlinear $\mathrm{THz}$ phenomena on silicon substrate are observed.When the $\mathrm{THz}$ electric field is increased from 2 to $100 \mathrm{kV} / \mathrm{cm}$ for single nano-gap SRRs, the resonance frequency is redshifted by $80 \mathrm{GHz}$ due to field-induced carrier multiplication in the silicon substrate in the nano-gaps, as illustrated in Figure 13. When the capacity cascade effect is taken into account and the nano-gap numbers are increased, the nonlinear effect can even be enlarged relatively. Additionally, via inter-valley scattering and auger recombination, strongfield $\mathrm{THz}$ can reduce the density of photocarriers injected by the optical pump. As a result, the approach advances to a new level by enabling the fabrication of enhanced nonlinear nano-/micro-composites for field-sensitive extreme $\mathrm{THz}$ nonlinear applications without the use of intense $\mathrm{THz}$ light sources.

In summary, the metal metasurface can be used to localize high fields, and the nonlinear phenomena are caused by transient material changes induced by the $\mathrm{THz}$ $E$-field. Both nonlinear mechanisms can contribute to ponderomotive energy, a non-resonant material regulation property, in both resonant and non-resonant metasurfaces. The resonant $\mathrm{THz}$ metasurface has not been implicated in the regulation of resonant materials, including coupling with molecular rotation and crystal lattice vibrations. Additionally, it was demonstrated that hot Dirac fermions can generate $\mathrm{THz}$ harmonics in graphene with high efficiency [132]. A few theoretical and experimental studies demonstrate that using graphene metasurfaces significantly improves $\mathrm{THz}$ harmonic generation [133, 134], and it is expected that experiments with topological surface states, such as Dirac and Weyl semi-metals [5, 135], will be reported. Zhao et al. demonstrated unequivocally that metasurfaces have advantages in nonlinear optics [136] and that these techniques could also be applied to nonlinear THz phenomena in topological surfaces. Metal metasurfaces can be deposited directly on Dirac or Weyl semi-metal surfaces to achieve $\mathrm{THz}$ nonlinearity via the metasurfaces' resonance localization effect [137]. Moreover, nonlinearity may be achieved by using strong $\mathrm{THz}$ pumping to the patterned Dirac or Weyl semi-metal material [137-139], viewed as the metasurface structure, such as

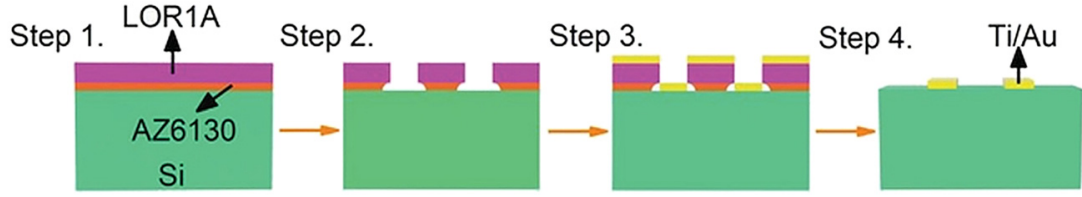

Step 8.
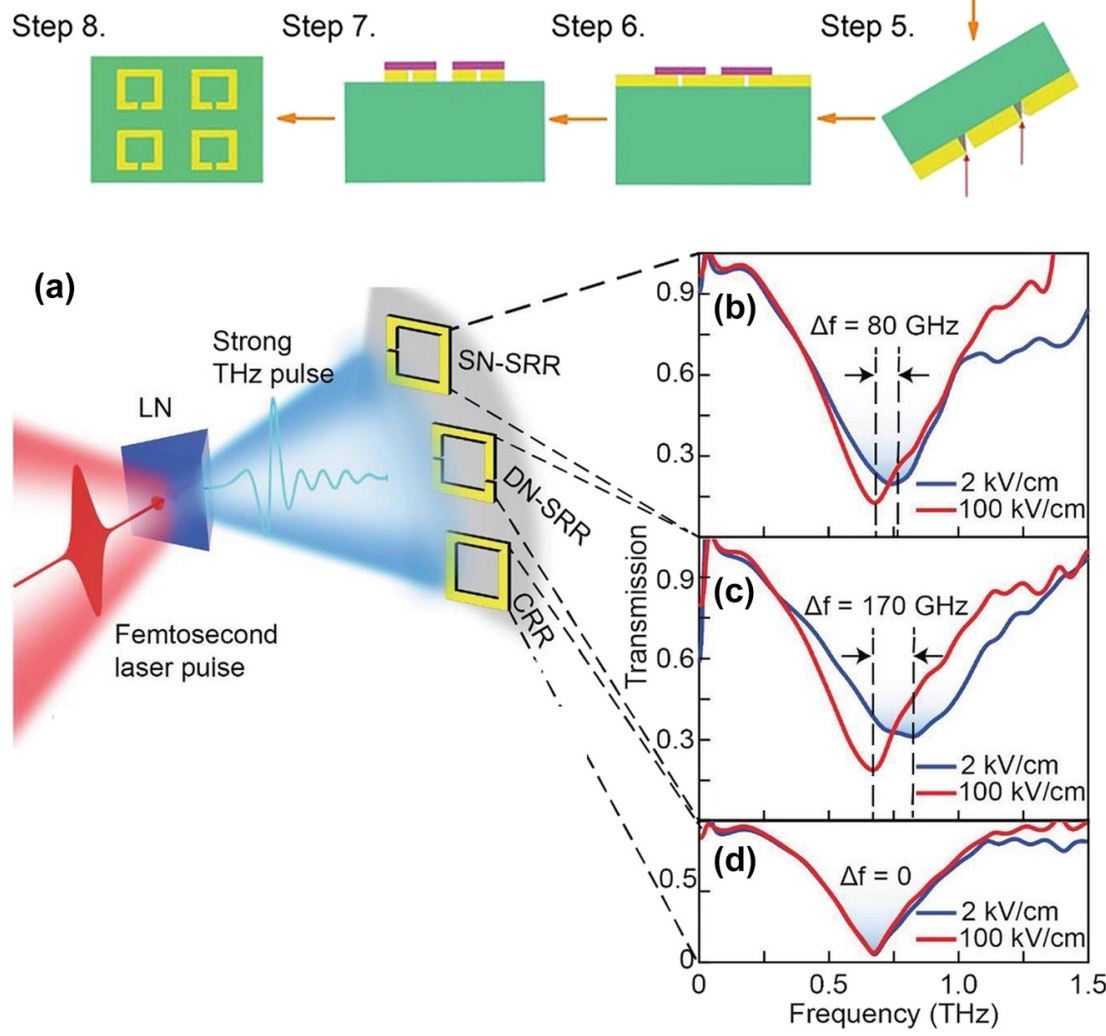

Figure 12: Fabrication technique for largescale $\mathrm{THz}$ metasurface with nano-gap. Reproduced with permission from Dong et al. [61] (C)Wiley-VCH GmbH (2021).

Figure 13: Nonlinear $\mathrm{THz}$ response for the THz SRR metasurface with nano-gaps.

(a) The strong field $\mathrm{THz}$ pulse is generated via titled wavefront technology in a lithium niobate crystal, and then is illuminated onto three different types of metasurfaces. Measured normalized transmitted spectra for (b) SN-SRRs, (c) DN-SRRs, and (d) CRRs under 2 and $100 \mathrm{kV} / \mathrm{cm}$ THz illuminations. Reproduced with permission from Dong et al. [61] (C)Wiley-VCH GmbH (2021). 
optical bound states in continuum. Metasurfaces, we anticipate, will allow for the realization of more novel nonlinear THz phenomena.

\section{Perspective}

The field strength of the international advanced THz light source is currently on the order of $\mathrm{MV} / \mathrm{cm}$, which is comparable to the electric field strength between semiconductor atoms and induces non-equilibrium states via electromagnetic field action and driving phonon coupling. If the field strength is increased further to $\mathrm{GV} / \mathrm{cm}$ ( 300 Tesla), it will be able to match the interatomic electric field in metals $(\sim 100 \mathrm{MV} / \mathrm{cm})$, and thus will be capable of inducing more extreme non-equilibrium states. By increasing the energy of a single pulse, implementing ultra-broadband emission, increasing the peak frequency, and shrinking the light spot, it is possible to achieve an electric field intensity of $\mathrm{GV} / \mathrm{cm}$, while nano-gap or nano-metal tip field enhancement may also be a method. In a strong field, the nonlinear effect is expected to realize multibody coupling and novel quantum states. Additionally, to meet the demand for material regulation, next-generation super-strong $\mathrm{THz}$ light sources must improve their pulse energy, field strength, polarization, and frequency tunability, which can be accomplished through the development of advanced $\mathrm{THz}$ source technology and the construction of more versatile $\mathrm{THz}$ metasurfaces. The integration of disciplines will significantly accelerate the development of extreme $\mathrm{THz}$ science and applications, and will truly benefit fundamental scientific research and potential applications in the future (Figure 14).

$\mathrm{THz}$ science and technology have advanced to unprecedented levels, demonstrating that $\mathrm{THz}$ electromagnetic radiation has evolved into a versatile frequency band for the discovery of novel physics and phenomena, as well as a powerful tool for scientific frontier research, particularly when coupled with other advanced measurement technologies in interdisciplinary research, such as THz pump-THz/optical/X-ray/ultrafast electron diffraction probe (see Figure 15).

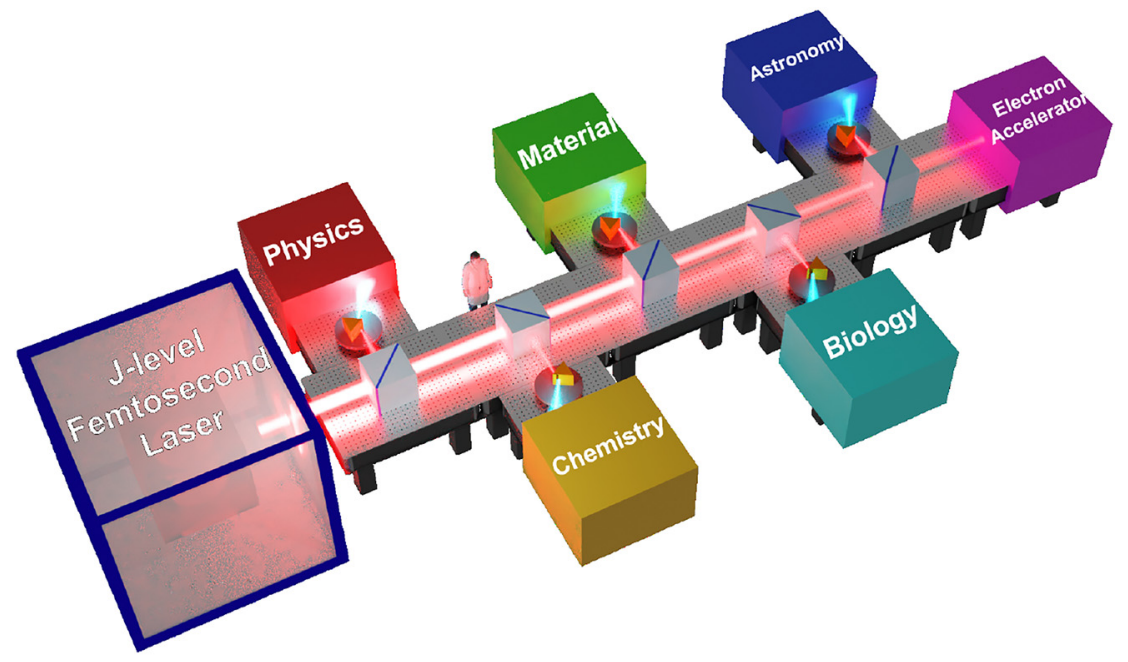

Figure 14: Intense THz sources enabled interdisciplinary applications.

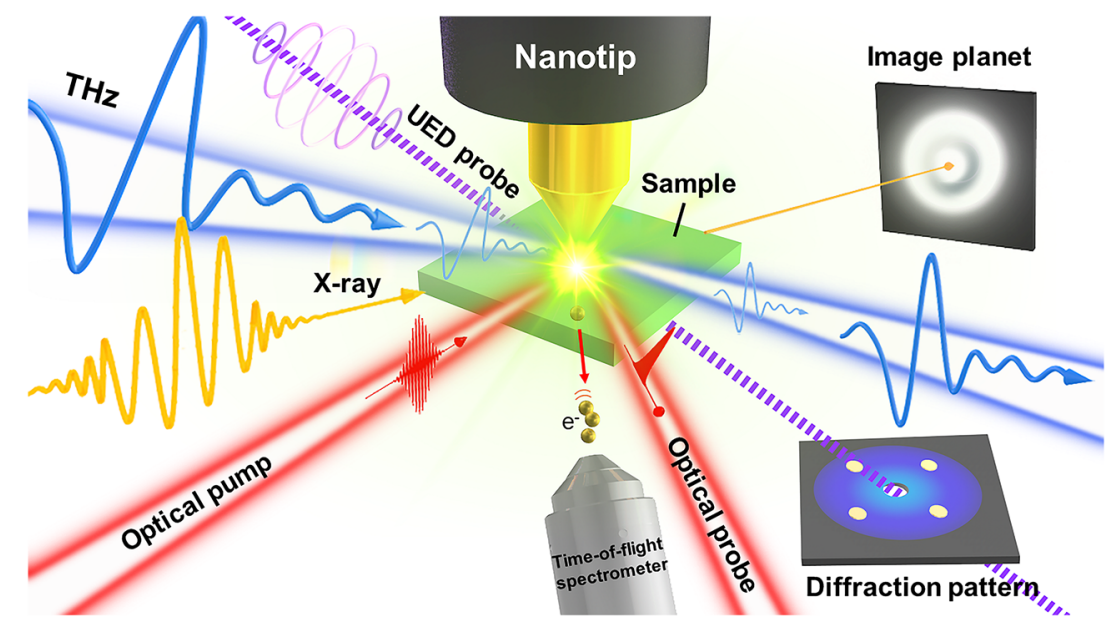

Figure 15: Strong-field THz coupled optical, UED, or X-ray probing system with nanophotonics methods. 
Acknowledgments: The authors thank Dr. Yaqing Zhang for the helpful discussion.

Author contribution: All authors contributed to the writing and revisions.

Research funding: Supported by the National Natural Science Foundation of China (61905007, 11827807, 62005140), the National Key R\&D Project (2019YFB2203102), the Open Project Program of Wuhan National Laboratory for Optoelectronics (2018WNLOKF001), and the Open Fund of Guangdong Provincial Key Laboratory of Information Photonics Technology (Guangdong University of Technology, No. GKPT20), China Postdoctoral Science Foundation (2020M670310).

Conflict of interest statement: The authors declare no conflicts of interest regarding this article.

\section{References}

[1] X. C. Zhang, A. Shkurinov, and Y. Zhang, "Extreme terahertz science," Nat. Photonics, vol. 11, pp. 16-18, 2017.

[2] F. Sekiguchi, H. Hirori, G. Yumoto, et al., "Enhancing the hot-phonon bottleneck effect in a metal halide perovskite by terahertz phonon excitation," Phys. Rev. Lett., vol. 126, p. 077401, 2021.

[3] L. Luo, D. Cheng, B. Song, et al., "A light-induced phononic symmetry switch and giant dissipationless topological photocurrent in ZrTe5," Nat. Mater., vol. 20, pp. 329-334, 2021.

[4] Q. Li, V. A. Stoica, M. Paściak, et al., "Subterahertz collective dynamics of polar vortices," Nature, vol. 592, pp. 376-380, 2021.

[5] C. P. Schmid, L. Weigl, P. Grössing, et al., "Tunable non-integer high-harmonic generation in a topological insulator," Nature, vol. 593, pp. 385-390, 2021.

[6] M. Plankl, P. E. Faria Junior, F. Mooshammer, et al., "Subcycle contact-free nanoscopy of ultrafast interlayer transport in atomically thin heterostructures," Nat. Photonics, vol. 15, pp. 594-600, 2021.

[7] C. Vaswani, M. Mootz, C. Sundahl, et al., "Terahertz second-harmonic generation from lightwave acceleration of symmetry-breaking nonlinear supercurrents," Phys. Rev. Lett., vol. 124, p. 207003, 2020.

[8] V. Balos, G. Bierhance, M. Wolf, and M. Sajadi, "Terahertz-magnetic-field induced ultrafast faraday rotation of molecular liquids," Phys. Rev. Lett., vol. 124, p. 093201 , 2020.

[9] S. Kovalev, R. M. A. Dantas, S. Germanskiy, et al., "Non-perturbative terahertz high-harmonic generation in the three-dimensional Dirac semimetal Cd3As2," Nat. Commun., vol. 11, p. 2451, 2020.

[10] J. Shi, E. Baldini, S. Latini, et al., "Room temperature terahertz electroabsorption modulation by excitons in monolayer transition metal dichalcogenides," Nano Lett., vol. 20, pp. 5214-5220, 2020.
[11] X. Li, T. Qiu, J. Zhang, et al., "Terahertz field-induced ferroelectricity in quantum paraelectric $\mathrm{SrTiO}_{3}$," Science, vol. 364, pp. 1079-1082, 2019.

[12] T. F. Nova, A. S. Disa, M. Fechner, and A. Cavalleri, "Metastable ferroelectricity in optically strained $\mathrm{SrTiO}_{3}$," Science, vol. 364, pp. 1075-1079, 2019.

[13] B. D. O’Shea, G. Andonian, S. K. Barber, et al., "Conductivity induced by high-field terahertz waves in dielectric material," Phys. Rev. Lett., vol. 123, p. 134801, 2019.

[14] E. Cinquanta, D. Meggiolaro, S. G. Motti, et al., "Ultrafast THz probe of photoinduced polarons in lead-halide perovskites," Phys. Rev. Lett., vol. 122, p. 166601, 2019.

[15] C. L. Johnson, B. E. Knighton, and J. A. Johnson, "Distinguishing nonlinear terahertz excitation pathways with two-dimensional spectroscopy," Phys. Rev. Lett., vol. 122, p. $073901,2019$.

[16] H. H. Fang, B. Han, C. Robert, et al., "Control of the exciton radiative lifetime in van der Waals heterostructures," Phys. Rev. Lett., vol. 123, p. 067401, 2019.

[17] X. Yang, C. Vaswani, C. Sundahl, et al., "Lightwave-driven gapless superconductivity and forbidden quantum beats by terahertz symmetry breaking," Nat. Photonics, vol. 13, pp. 707-713, 2019.

[18] L. Luo, X. Yang, X. Liu, et al., “Ultrafast manipulation of topologically enhanced surface transport driven by mid-infrared and terahertz pulses in Bi2Se3," Nat. Commun., vol. 10, p. 607, 2019.

[19] J. Reimann, S. Schlauderer, C. P. Schmid, et al., "Subcycle observation of lightwave-driven Dirac currents in a topological surface band," Nature, vol. 562, pp. 396-400, 2018.

[20] C. Vicario, M. Shalaby, and C. P. Hauri, "Subcycle extreme nonlinearities in GaP induced by an ultrastrong terahertz field," Phys. Rev. Lett., vol. 118, p. 083901, 2017.

[21] T. Kampfrath, K. Tanaka, and K. A. Nelson, "Resonant and nonresonant control over matter and light by intense terahertz transients," Nat. Photonics, vol. 7, pp. 680-690, 2013.

[22] R. Damari, O. Weinberg, D. Krotkov, et al., "Strong coupling of collective intermolecular vibrations in organic materials at terahertz frequencies," Nat. Commun., vol. 10, p. 3248, 2019.

[23] E. J. Sie, C. M. Nyby, C. D. Pemmaraju, et al., "An ultrafast symmetry switch in a Weyl semimetal," Nature, vol. 565, pp. 61-66, 2019.

[24] X. Chai, X. Ropagnol, S. M. Raeis-Zadeh, M. Reid, S. Safavi-Naeini, and T. Ozaki, "Subcycle terahertz nonlinear optics," Phys. Rev. Lett., vol. 121, p. 143901, 2018.

[25] A. X. Gray, M. C. Hoffmann, J. Jeong, et al., "Ultrafast terahertz field control of electronic and structural interactions in vanadium dioxide," Phys. Rev. B, vol. 98, p. 045104, 2018.

[26] S. Fleischer, Y. Zhou, R. W. Field, and K. A. Nelson, "Molecular orientation and alignment by intense single-cycle $\mathrm{THz}$ pulses," Phys. Rev. Lett., vol. 107, p. 163603, 2011.

[27] K. Kitano, N. Ishii, N. Kanda, et al., "Orientation of jet-cooled polar molecules with an intense single-cycle THz pulse," Phys. Rev., vol. 88, p. 061405, 2013.

[28] Z.-Y. Zhao, Y.-C. Han, Y. Huang, and S.-L. Cong, "Field-free orientation by a single-cycle $\mathrm{THz}$ pulse: the $\mathrm{Nal}$ and $\mathrm{IBr}$ molecules,"J. Chem. Phys., vol. 139, p. 044305, 2013. 
[29] K. N. Egodapitiya, S. Li, and R. R. Jones, "Terahertz-induced field-free orientation of rotationally excited molecules," Phys. Rev. Lett., vol. 112, p. 103002, 2014.

[30] S. Schlauderer, C. Lange, S. Baierl, et al., "Temporal and spectral fingerprints of ultrafast all-coherent spin switching," Nature, vol. 569, pp. 383-387, 2019.

[31] S. Baierl, J. H. Mentink, M. Hohenleutner, et al., "Terahertz-driven nonlinear spin response of antiferromagnetic nickel oxide," Phys. Rev. Lett., vol. 117, p. 197201, 2016.

[32] R. V. Mikhaylovskiy, T. J. Huisman, R. V. Pisarev, T. Rasing, and A. V. Kimel, "Selective excitation of terahertz magnetic and electric dipoles in Er3+ ions by femtosecond laser pulses in ErFe03," Phys. Rev. Lett., vol. 118, p. 017205, 2017.

[33] P. Němec, M. Fiebig, T. Kampfrath, and A. V. Kimel, “Antiferromagnetic opto-spintronics," Nat. Phys., vol. 14, pp. 229-241, 2018.

[34] D. Afanasiev, J. R. Hortensius, B. A. Ivanov, et al., "Ultrafast control of magnetic interactions via light-driven phonons," Nat. Mater., vol. 20, pp. 607-611, 2021.

[35] P. Vaidya, S. A. Morley, J. van Tol, et al., "Subterahertz spin pumping from an insulating antiferromagnet," Science, vol. 368, pp. 160-165, 2020.

[36] E. A. Mashkovich, K. A. Grishunin, R. V. Mikhaylovskiy, et al., "Terahertz optomagnetism: nonlinear $\mathrm{THz}$ excitation of $\mathrm{GHz}$ spin waves in antiferromagnetic FeB03," Phys. Rev. Lett., vol. 123, p. 157202, 2019.

[37] T. L. Cocker, V. Jelic, R. Hillenbrand, and F. A. Hegmann, "Nanoscale terahertz scanning probe microscopy," Nat. Photonics, vol. 15, pp. 558-569, 2021.

[38] D. Peller, L. Z. Kastner, T. Buchner, et al., "Sub-cycle atomic-scale forces coherently control a single-molecule switch," Nature, vol. 585, pp. 58-62, 2020.

[39] V. Jelic, K. Iwaszczuk, P. H. Nguyen, et al., "Ultrafast terahertz control of extreme tunnel currents through single atoms on a silicon surface," Nat. Phys., vol. 13, pp. 591-598, 2017.

[40] K. Yoshioka, I. Katayama, Y. Minami, et al., "Real-space coherent manipulation of electrons in a single tunnel junction by single-cycle terahertz electric fields," Nat. Photonics, vol. 10, pp. 762-765, 2016

[41] T. L. Cocker, V. Jelic, M. Gupta, et al., "An ultrafast terahertz scanning tunnelling microscope," Nat. Photonics, vol. 7, pp. 620-625, 2013.

[42] H. Tang, L. Zhao, P. Zhu, et al., "Stable and scalable multistage terahertz-driven particle accelerator," Phys. Rev. Lett., vol. 127, p. 074801, 2021.

[43] H. Xu, L. Yan, Y. Du, et al., “Cascaded high-gradient terahertz-driven acceleration of relativistic electron beams," Nat. Photonics, vol. 15, pp. 426-430, 2021.

[44] D. Zhang, M. Fakhari, H. Cankaya, A.-L. Calendron, N. H. Matlis, and F. X. Kärtner, "Cascaded multicycle terahertz-driven ultrafast electron acceleration and manipulation," Phys. Rev. X, vol. 10, p. 011067, 2020.

[45] M. T. Hibberd, A. L. Healy, D. S. Lake, et al., "Acceleration of relativistic beams using laser-generated terahertz pulses," Nat. Photonics, vol. 14, pp. 755-759, 2020.

[46] L. Zhao, Z. Wang, H. Tang, et al., "Terahertz oscilloscope for recording time information of ultrashort electron beams," Phys. Rev. Lett., vol. 122, p. 144801, 2019.
[47] D. Zhang, A. Fallahi, M. Hemmer, et al., "Femtosecond phase control in high-field terahertz-driven ultrafast electron sources," Optica, vol. 6, p. 872, 2019.

[48] D. Zhang, A. Fallahi, M. Hemmer, et al., "Segmented terahertz electron accelerator and manipulator (STEAM)," Nat. Photonics, vol. 12, pp. 336-342, 2018.

[49] C. Kealhofer, W. Schneider, D. Ehberger, A. Ryabov, F. Krausz, and P. Baum, "All-optical control and metrology of electron pulses," Science, vol. 352, pp. 429-433, 2016.

[50] W. Ronny Huang, A. Fallahi, X. Wu, et al., "Terahertz-driven, all-optical electron gun," Optica, vol. 3, p. 1209, 2016.

[51] W. R. Huang, E. A. Nanni, K. Ravi, et al., "Toward a terahertz-driven electron gun,” Sci. Rep., vol. 5, p. 14899, 2015.

[52] E. A. Nanni, W. R. Huang, K.-H. Hong, et al., "Terahertz-driven linear electron acceleration,” Nat. Commun., vol. 6, p. 8486, 2015.

[53] L. Wimmer, G. Herink, D. R. Solli, S. V. Yalunin, K. E. Echternkamp, and C. Ropers, "Terahertz control of nanotip photoemission,” Nat. Phys., vol. 10, pp. 432-436, 2014.

[54] Y. Li, C. Chang, Z. Zhu, L. Sun, and C. Fan, "Terahertz wave enhances permeability of the voltage-gated calcium channel,"J. Am. Chem. Soc., vol. 143, pp. 4311-4318, 2021.

[55] T. Tachizaki, R. Sakaguchi, S. Terada, K.-I. Kamei, and H. Hirori, "Terahertz pulse-altered gene networks in human induced pluripotent stem cells,” Opt Lett., vol. 45, p. 6078, 2020.

[56] S. Yamazaki, M. Harata, T. Idehara, et al., "Actin polymerization is activated by terahertz irradiation," Sci. Rep., vol. 8, p. 9990, 2018.

[57] K. Fan, H. Y. Hwang, M. Liu, et al., "Nonlinear terahertz metamaterials via field-enhanced carrier dynamics in GaAs," Phys. Rev. Lett., vol. 110, p. 217404, 2013.

[58] A. Jooshesh, V. Bahrami-Yekta, J. Zhang, T. Tiedje, T. E. Darcie, and R. Gordon, "Plasmon-enhanced below bandgap photoconductive terahertz generation and detection," Nano Lett., vol. 15, pp. 8306-8310, 2015.

[59] C. Lange, T. Maag, M. Hohenleutner, et al., "Extremely nonperturbative nonlinearities in GaAs driven by atomically strong terahertz fields in gold metamaterials," Phys. Rev. Lett., vol. 113, p. 227401, 2014.

[60] X. Zhao, J. Zhang, K. Fan, et al., "Nonlinear terahertz metamaterial perfect absorbers using GaAs [Invited]," Photon. Res., vol. 4, p. A16, 2016.

[61] T. Dong, S. Li, M. Manjappa, et al., "Nonlinear THz-nano metasurfaces," Adv. Funct. Mater., vol. 31, p. 2100463, 2021.

[62] S. G. Rodrigo and L. Martín-Moreno, "Absorption-induced transparency metamaterials in the terahertz regime," Opt. Lett., vol. 41, p. 293, 2016.

[63] A. T. Tarekegne, H. Hirori, K. Tanaka, K. Iwaszczuk, and P. U. Jepsen, "Impact ionization dynamics in silicon by MV/cm THz fields," New J. Phys., vol. 19, p. 123018, 2017.

[64] J. Zhang, X. Zhao, K. Fan, et al., "Terahertz radiation-induced sub-cycle field electron emission across a split-gap dipole antenna," Appl. Phys. Lett., vol. 107, p. 231101, 2015.

[65] S. Han, J.-Y. Kim, T. Kang, et al., "Colossal terahertz nonlinearity in angstrom- and nanometer-sized gaps," ACS Photonics, vol. 3, pp. 1440-1445, 2016. 
[66] G. Herink, L. Wimmer, and C. Ropers, "Field emission at terahertz frequencies: AC-tunneling and ultrafast carrier dynamics," New J. Phys., vol. 16, p. 123005, 2014.

[67] D.-S. Kim, Y.-M. Bahk, J.-Y. Kim, et al., "Terahertz funneling-induced quantum tunneling at angstrom scale," in 2016 41st International Conference on Infrared, Millimeter, and Terahertz Waves (IRMMW-THz), Copenhagen, Denmark, IEEE, Sept. 2016, pp. 1-3.

[68] J.-Y. Kim, B. J. Kang, J. Park, et al., "Terahertz quantum plasmonics of nanoslot antennas in nonlinear regime," Nano Lett., vol. 15, pp. 6683-6688, 2015.

[69] K. Yoshioka, Y. Minami, K.-i. Shudo, et al., "Terahertz-field-induced nonlinear electron delocalization in Au nanostructures," Nano Lett., vol. 15, pp. 1036-1040, 2015.

[70] B. C. Pein, W. Chang, H. Y. Hwang, et al., "Terahertz-driven luminescence and colossal Stark effect in CdSe-CdS colloidal quantum dots," Nano Lett., vol. 17, pp. 5375-5380, 2017.

[71] I. Katayama, H. Aoki, J. Takeda, et al., "Ferroelectric soft mode in a SrTiO 3 thin film impulsively driven to the anharmonic regime using intense picosecond terahertz pulses," Phys. Rev. Lett., vol. 108, p. 097401, 2012.

[72] S. W. Jolly, N. H. Matlis, F. Ahr, et al., "Spectral phase control of interfering chirped pulses for high-energy narrowband terahertz generation," Nat. Commun., vol. 10, p. 2591, 2019.

[73] A. D. Koulouklidis, C. Gollner, V. Shumakova, et al., "Observation of extremely efficient terahertz generation from mid-infrared two-color laser filaments," Nat. Commun., vol. 11, p. 292, 2020.

[74] G. Liao, Y. Li, H. Liu, et al., "Multimillijoule coherent terahertz bursts from picosecond laser-irradiated metal foils," Proc. Natl. Acad. Sci. U. S. A., vol. 116, pp. 3994-3999, 2019.

[75] J. Lu, S.-H. Lee, X. Li, et al., "Efficient terahertz generation in highly nonlinear organic crystal HMB-TMS," Opt. Express, vol. 26, p. 30786, 2018.

[76] J. A. Fülöp, S. Tzortzakis, and T. Kampfrath, "Laser-driven strong-field terahertz sources," Adv. Opt. Mater., vol. 8, p. 1900681, 2020.

[77] Y. Tian, J. Liu, Y. Bai, et al., "Femtosecond-laser-driven wire-guided helical undulator for intense terahertz radiation," Nat. Photonics, vol. 11, pp. 242-246, 2017.

[78] I. Dey, K. Jana, V. Y. Fedorov, et al., "Highly efficient broadband terahertz generation from ultrashort laser filamentation in liquids," Nat. Commun., vol. 8, p. 1184, 2017.

[79] B. Zhang, Z. Ma, J. Ma, et al., "1.4-mJ high energy terahertz radiation from lithium niobates," Laser Photon. Rev., vol. 15, p. 2000295, 2021.

[80] X. Chen, H. Wang, C. Wang, et al., "Efficient generation and arbitrary manipulation of chiral terahertz waves emitted from $\mathrm{Bi}_{2} \mathrm{Te}_{3}-\mathrm{Fe}$ heterostructures," Adv. Photo. Res., vol. 2, p. 2000099, 2021.

[81] H. Zhao, X. Chen, C. Ouyang, et al., "Generation and manipulation of chiral terahertz waves in the three-dimensional topological insulator Bi2Te3," Adv. Photonics, vol. 2, pp. 066003-066003, 2020.

[82] F. Kärtner, F. Ahr, A.-L. Calendron, et al., “AXSIS: exploring the frontiers in attosecond $\mathrm{X}$-ray science, imaging and spectroscopy," Nucl. Instrum. Methods Phys. Res. Sect. A
Accel. Spectrom. Detect. Assoc. Equip., vol. 829, pp. 24-29, 2016.

[83] K. Y. Kim and Y. S. You, “Terahertz-driven harmonics," Nat. Photonics, vol. 8, pp. 92-94, 2014.

[84] M. Liu, H. Y. Hwang, H. Tao, et al., "Terahertz-field-induced insulator-to-metal transition in vanadium dioxide metamaterial," Nature, vol. 487, pp. 345-348, 2012.

[85] S.-W. Huang, E. Granados, W. R. Huang, K.-H. Hong, L. E. Zapata, and F. X. Kärtner, "High conversion efficiency, high energy terahertz pulses by optical rectification in cryogenically cooled lithium niobate," Opt. Lett., vol. 38, p. 796, 2013.

[86] C. Vicario, A. V. Ovchinnikov, S. I. Ashitkov, M. B. Agranat, V. E. Fortov, and C. P. Hauri, "Generation of 09-mJ THz pulses in DSTMS pumped by a Cr:mg_2SiO_4 laser,” Opt. Lett., vol. 39, p. 6632, 2014.

[87] G. Polónyi, B. Monoszlai, G. Gäumann, et al., “High-energy terahertz pulses from semiconductors pumped beyond the three-photon absorption edge," Opt. Express, vol. 24, no. 21, p. 23872, 2016.

[88] N. Stojanovic and M. Drescher, "Accelerator- and laser-based sources of high-field terahertz pulses," J. Phys. B Atom. Mol. Opt. Phys., vol. 46, no. 19, p. 192001, 2013.

[89] X. Ropagnol, Z. Kovács, B. Gilicze, et al., “Intense sub-terahertz radiation from wide-bandgap semiconductor based large-aperture photoconductive antennas pumped by UV lasers," New J. Phys., vol. 21, no. 11, p. 113042, 2019.

[90] N. T. Yardimci, S.-H. Yang, C. W. Berry, and M. Jarrahi, "High-power terahertz generation using large-area plasmonic photoconductive emitters," IEEE Trans. Terahertz Sci. Technol., vol. 5, no. 2, pp. 223-229, 2015.

[91] A. Gopal, P. Singh, S. Herzer, et al., "Characterization of $700 \mu$ J T rays generated during high-power laser solid interaction," Opt. Lett., vol. 38, no. 22, p. 4705, 2013.

[92] G.-Q. Liao, H. Liu, G. G. Scott, et al., "Towards terawatt-scale spectrally tunable terahertz pulses via relativistic laser-foil interactions," Phys. Rev. X, vol. 10, no. 3, p. 031062 , 2020.

[93] Q. Jin, E. Yiwen, K. Williams, J. Dai, and X.-C. Zhang, "Observation of broadband terahertz wave generation from liquid water,” Appl. Phys. Lett., vol. 111, no. 7, p. 071103 , 2017.

[94] T. Kampfrath, M. Battiato, P. Maldonado, et al., "Terahertz spin current pulses controlled by magnetic heterostructures," Nat. Nanotechnol., vol. 8, pp. 256-260, 2013.

[95] T. J. Huisman, R. V. Mikhaylovskiy, J. D. Costa, et al., "Femtosecond control of electric currents in metallic ferromagnetic heterostructures," Nat. Nanotechnol., vol. 11, pp. 455-458, 2016.

[96] Y. Wu, M. Elyasi, X. Qiu, et al., "High-performance THz emitters based on ferromagnetic/nonmagnetic heterostructures," Adv. Mater., vol. 29, p. 1603031, 2017.

[97] C. Zhou, Y. P. Liu, Z. Wang, et al., "Broadband terahertz generation via the interface inverse rashba-edelstein effect," Phys. Rev. Lett., vol. 121, p. 086801, 2018.

[98] B. Monoszlai, C. Vicario, M. Jazbinsek, and C. P. Hauri, "High-energy terahertz pulses from organic crystals: DAST and DSTMS pumped at Ti:sapphire wavelength," Opt. Lett., vol. 38, p. 5106, 2013. 
[99] C. Ruchert, C. Vicario, and C. P. Hauri, "Spatiotemporal focusing dynamics of intense supercontinuum THz pulses," Phys. Rev. Lett., vol. 110, p. 123902, 2013.

[100] C. Vicario, B. Monoszlai, and C. P. Hauri, "GV/m single-cycle terahertz fields from a laser-driven large-size partitioned organic crystal," Phys. Rev. Lett., vol. 112, p. 213901, 2014.

[101] K. H. Yang, P. L. Richards, and Y. R. Shen, "Generation of far-infrared radiation by picosecond light pulses in $\mathrm{LiNbO}_{3}$," Appl. Phys. Lett., vol. 19, pp. 320-323, 1971.

[102] J. Hebling, G. Almasi, I. Kozma, and J. Kuhl, "Velocity matching by pulse front tilting for large area THz-pulse generation," Opt Express, vol. 10, p. 1161, 2002.

[103] A. G. Stepanov, J. Hebling, and J. Kuhl, "Efficient generation of subpicosecond terahertz radiation by phase-matched optical rectification using ultrashort laser pulses with tilted pulse fronts," Appl. Phys. Lett., vol. 83, pp. 3000-3002, 2003.

[104] K.-L. Yeh, M. C. Hoffmann, J. Hebling, and K. A. Nelson, "Generation of $10 \mu$ J ultrashort terahertz pulses by optical rectification,” Appl. Phys. Lett., vol. 90, p. 171121, 2007.

[105] F. Blanchard, L. Razzari, H. Bandulet, et al., "Generation of $1.5 \mu$ single-cycle terahertz pulses by optical rectification from a large aperture ZnTe crystal," Opt. Express, vol. 15, no. 20, p. 13212, 2007.

[106] M. C. Hoffmann, K.-L. Yeh, J. Hebling, and K. A. Nelson, "Efficient terahertz generation by optical rectification at 1035nm," Opt. Express, vol. 15, no. 18, p. 11706, 2007.

[107] A. G. Stepanov, L. Bonacina, S. V. Chekalin, and J.-P. Wolf, "Generation of $30 \mu$ J single-cycle terahertz pulses at $100 \mathrm{~Hz}$ repetition rate by optical rectification," Opt. Lett., vol. 33, p. 2497, 2008.

[108] H. Hirori, A. Doi, F. Blanchard, and K. Tanaka, "Single-cycle terahertz pulses with amplitudes exceeding $1 \mathrm{MV} / \mathrm{cm}$ generated by optical rectification in LiNb03," Appl. Phys. Lett., vol. 98, p. 091106, 2011.

[109] A. G. Stepanov, L. Bonacina, S. V. Chekalin, and J.-P. Wolf, "Generation of $30 \mu$ j single-cycle terahertz pulses at $100 \mathrm{hz}$ repetition rate by optical rectification," Opt. Lett., vol. 33, pp. 2497-2499, 2008.

[110] C. Vicario, B. Monoszlai, C. Lombosi, et al., "Pump pulse width and temperature effects in lithium niobate for efficient THz generation," Opt. Lett., vol. 38, p. 5373, 2013.

[111] J. A. Fülöp, L. Pálfalvi, S. Klingebiel, et al., "Generation of sub-mJ terahertz pulses by optical rectification," Opt. Lett., vol. 37, p. 557, 2012.

[112] X. Wu, S. Carbajo, K. Ravi, et al., "Terahertz generation in lithium niobate driven by Ti:sapphire laser pulses and its limitations," Opt. Lett., vol. 39, p. 5403, 2014.

[113] X. Wu, A.-L. Calendron, K. Ravi, et al., "Optical generation of single-cycle $10 \mathrm{MW}$ peak power $100 \mathrm{GHz}$ waves," Opt. Express, vol. 24, p. 21059, 2016.

[114] J. A. Fülöp, G. Polónyi, B. Monoszlai, et al., "Highly efficient scalable monolithic semiconductor terahertz pulse source," Optica, vol. 3, p. 1075, 2016.

[115] B. K. Ofori-Okai, P. Sivarajah, W. Ronny Huang, and K. A. Nelson, "THz generation using a reflective stair-step echelon,” Opt. Express, vol. 24, p. 5057, 2016.

[116] S.-C. Zhong, J. Li, Z.-H. Zhai, et al., "Generation of 0.19-mj thz pulses in linbo3 driven by $800-\mathrm{nm}$ femtosecond laser," Opt. Express, vol. 24, pp. 14828-14835, 2016.
[117] F. Blanchard, X. Ropagnol, H. Hafez, et al., "Effect of extreme pump pulse reshaping on intense terahertz emission in lithium niobate at multimillijoule pump energies," Opt. Lett., vol. 39, p. 4333, 2014.

[118] X. Wu, C. Zhou, W. R. Huang, F. Ahr, and F. X. Kärtner, "Temperature dependent refractive index and absorption coefficient of congruent lithium niobate crystals in the terahertz range," Opt. Express, vol. 23, p. 29729, 2015.

[119] X.-j. Wu, J.-I. Ma, B.-l. Zhang, et al., "Highly efficient generation of $0.2 \mathrm{~m}$ J terahertz pulses in lithium niobate at room temperature with sub-50 fs chirped Ti:sapphire laser pulses," Opt. Express, vol. 26, p. 7107, 2018.

[120] J. A. Fülöp, L. Pálfalvi, M. C. Hoffmann, and J. Hebling, "Towards generation of mj-level ultrashort thz pulses by optical rectification," Opt. Express, vol. 19, pp. 15090-15097, 2011.

[121] K. Ravi, W. R. Huang, S. Carbajo, X. Wu, and F. Kärtner, "Limitations to $\mathrm{THz}$ generation by optical rectification using tilted pulse fronts," Opt. Express, vol. 22, p. 20239, 2014.

[122] K. Ravi, W. R. Huang, S. Carbajo, et al., "Theory of terahertz generation by optical rectification using tilted-pulse-fronts," Opt. Express, vol. 23, p. 5253, 2015.

[123] L. Wang, T. Kroh, N. H. Matlis, and F. Kärtner, "Full 3d + 1 modeling of tilted-pulse-front setups for single-cycle terahertz generation," J. Opt. Soc. Am. B, vol. 37, pp. 1000-1007, 2020.

[124] L. Wang, G. Tóth, J. Hebling, and F. Kärtner, "Tilted-pulse-front schemes for terahertz generation," Laser Photon. Rev., vol. 14, p. 2000021, 2020.

[125] J. A. Porto, F. J. García-Vidal, and J. B. Pendry, "Transmission resonances on metallic gratings with very narrow slits," Phys. Rev. Lett., vol. 83, pp. 2845-2848, 1999.

[126] S. Kang, Z. Qian, V. Rajaram, S. D. Calisgan, A. Alù, and M. Rinaldi, "Ultra-Narrowband metamaterial absorbers for high spectral resolution infrared spectroscopy," Adv. Opt. Mater., vol. 7, 2019, p. 1801236. https://doi.org/10.1002/ adom.201801236.

[127] D. Ji, A. Cheney, N. Zhang, et al., "Efficient mid-infrared light confinement within sub-5-nm gaps for extreme field enhancement," Adv. Opt. Mater., vol. 5, p. 1700223, 2017.

[128] H. R. Seren, J. Zhang, G. R. Keiser, et al., "Nonlinear terahertz devices utilizing semiconducting plasmonic metamaterials," Light Sci. Appl., vol. 5, pp. e16078, 2016.

[129] G. R. Keiser, N. Karl, P. Q. Liu, et al., "Nonlinear terahertz metamaterials with active electrical control," Appl. Phys. Lett., vol. 111, p. 121101, 2017.

[130] Y.-M. Bahk, B. J. Kang, Y. S. Kim, et al., “Electromagnetic saturation of angstrom-sized quantum barriers at terahertz frequencies," Phys. Rev. Lett., vol. 115, p. 125501, 2015.

[131] X. Chen, "Atomic layer lithography of wafer-scale nanogap arrays for extreme confinement of electromagnetic waves," Nat. Commun., vol. 4, no. 1, p. 2631, 2013.

[132] H. A. Hafez, S. Kovalev, J.-C. Deinert, et al., "Extremely efficient terahertz high-harmonic generation in graphene by hot Dirac fermions," Nature, vol. 561, pp. 507-511, 2018.

[133] J. D. Cox, A. Marini, and F. J. G. de Abajo, "Plasmon-assisted high-harmonic generation in graphene," Nat. Commun., vol. 8, p. 14380, 2017. 
[134] J.-C. Deinert, D. Alcaraz Iranzo, R. Pérez, et al., “Gratinggraphene metamaterial as a platform for terahertz nonlinear Photonics," ACS Nano, vol. 15, pp. 1145-1154, 2021.

[135] Y. Bai, F. Fei, S. Wang, et al., "High-harmonic generation from topological surface states," Nat. Phys., vol. 17, pp. 311-315, 2021.

[136] Y. Zhao, Y. Yang, and H.-B. Sun, "Nonlinear meta-optics towards applications," PhotoniX, vol. 2, p. 3, 2021.
[137] Q. Zhang, G. Hu, W. Ma, et al., “Interface nano-optics with van der Waals polaritons,” Nature, vol. 597, pp. 187-195, 2021.

[138] D. Hu, H. Li, Y. Zhu, et al., "Ultra-sensitive nanometric flat laser prints for binocular stereoscopic image," Nat. Commun., vol. 12, p. 1154, 2021.

[139] F. Qin, B. Liu, L. Zhu, et al., “ $\pi$-phase modulated monolayer supercritical lens,” Nat. Commun., vol. 12, p. 32, 2021. 